\title{
Synthesis and biological evaluation of novel substituted pyrrolo[2,3-d]pyrimidines as anticancer agents
}

\author{
Sandip P. Dholakia ${ }^{1 *}$, Kaushik B. Kanada ${ }^{2}$, Ghanshyam R. Parmar ${ }^{3}$ \\ ${ }^{1}$ Department of Chemistry, Shankersinh Vaghela Bapu Institute of Pharmacy, Gandhinagar, Gujarat, India. \\ ${ }^{2}$ Department of Chemistry, Saraswati Institute of Pharmaceutical Education \& Research, Gandhinagar, Gujarat, India. \\ ${ }^{3}$ Department of Pharmacy, Sumandeep Vidyapeeth, Vadodara, Gujarat, India.
}

\author{
ARTICLE INFO \\ Received on: 22/11/2019 \\ Accepted on: 15/04/2020 \\ Available online: 05/06/2020
Key words:
Doxorubicin, pyrrolo[2,3-d]
pyrimidine, anticancer, breast
cancer.

\begin{abstract}
Pyrrolopyrimidines are well known scaffold, which play a critical role as anticancer agents, so it thought of interest to synthesize a series of novel substituted pyrrolo[2,3- $d$ ]pyrimidines having diverse groups at position C4 and N7 of the pyrrolo[2,3- $d$ ]pyrimidine core and performed in vitro screening against MDA-MB-468 (breast cancer cell line) cell line. The details of the synthetic methods and characterization data of the synthesized compounds have been presented in this study. Compounds $8 \mathrm{a}, \mathbf{8 h}, \mathbf{8 j}, \mathbf{9 h}, \mathbf{9 i}, \mathbf{9 j}, \mathbf{9 m}, \mathbf{9 n}$, and 90 showed the excellent anticancer activity compared to standard doxorubicin with an $\mathrm{IC}_{50}$ value of $6.17 \mu \mathrm{M} / \mathrm{ml}$ against MDA-MB-468 (breast cancer cell line), which was non-toxic to normal vero cell line.
\end{abstract}

\section{INTRODUCTION}

Cancer is characterized by the uncontrolled growth of abnormal cells (Grange et al., 2002; Kari and Dave, 2012). The abnormal growth can also be referred to as a neoplasm. Neoplasms can be benign (noncancerous) or malignant (cancerous). Cancerous growths can occur in any organ of the body and are characterized by three distinct properties: the cells replicate rapidly with reduced growth control, the cells lose contact inhibition in vitro, and the resulting neoplasm invades surrounding tissues and may spread to other parts of the body (Foye et al., 1995; Kleinsmith, 2006). The cells of benign tumors have reduced growth control but do not invade surrounding tissues or spread to other parts of the body. Other important aspects of cancer cells include the ability to be self-sufficient and generate local angiogenesis while resisting antigrowth and apoptosis signals. If the spread of a malignant neoplasm is not controlled, it can result in death (Hadfield et al.,

${ }^{*}$ Corresponding Author

Sandip P. Dholakia, Department of Chemistry, Shankersinh Vaghela Bapu Institute of Pharmacy, Gandhinagar, Gujarat.

E-mail: sandip.chemistry2008@gmail.com
2003; Honore et al., 2005; Jordan et al., 1998; Whitman et al., 1995).

Most breast cancers begin either in the breast tissue made up of glands for milk production, called lobules, or in the ducts that connect the lobules to the nipple. The remainder of the breast is made up of fatty, connective, and lymphatic tissues (Edge et al., 2010).

In 2016, an estimated 252,710 new cases of invasive breast cancer will be diagnosed among women and 2,470 cases will be diagnosed in men. Besides, 63,410 cases of in situ breast carcinoma will be diagnosed among women. Approximately 40,610 women and 460 men are expected to die from breast cancer in 2016 (Miller et al., 2016).

An increasing interest in the biological studies of pyrrolo[2,3- $d]$ pyrimidine in the past decade is a consequence of their wide usage as a pharmaceutically important class of compounds. Pyrrolo[2,3- $d]$ pyrimidine derivatives have a considerable potential in the field of chemotherapy as they were found to exhibit their antitumor activity by inhibiting different types of enzymes such as cyclin-dependent kinase, $\operatorname{Src}$ and Abl tyrosine kinase, glycogen synthase kinase-3, adenosine deaminase, and epidermal growth factor receptor protein tyrosine kinase (Ghorab et al., 2010). The derivatives of pyrrolo[2,3-d] 
pyrimidine have already been discovered as antitumor agents by the NCI (National Cancer Institute, Bethesda, MD) on HCT116 and other cell lines. Some pyrrolo[2,3-d]pyrimidines(1) structurally related with allopurinol (2), 6-mercaptopurine (3), and 1- (1, 1-dimethylethyl)- 3- (1-naphthalenyl)- $1 H$-pyrazolo[3,4- $d]$ pyrimidin-4-amine (1-NA-PP1) (4) have also been reported as potent inhibitors for the growth of several human tumor cell lines (Gangjee et al., 2010).

In view of these observations, we undertook the synthesis of three series of novel pyrrolo[2,3- $d]$ pyrimidine analogs (Scheme $1)$ and in vitro screening on breast cancer cell line (8-10).<smiles>C=Cc1c(C)cccc1-c1nn(C(C)(C)C)c2ncnc(N)c12</smiles>

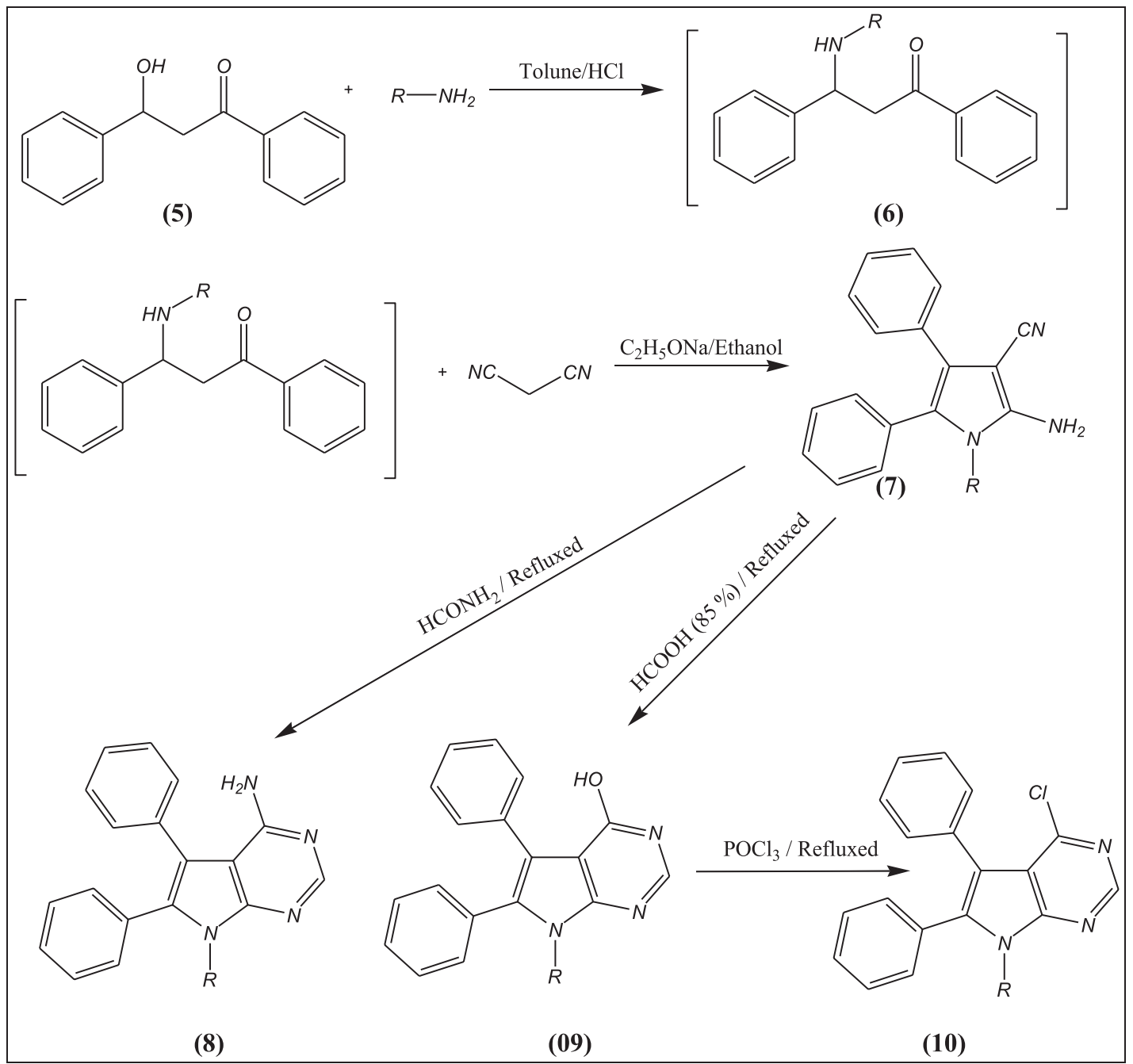

Scheme 1. Synthesis of pyrrolo[2,3-d]pyrimidine analogs. 


\section{MATERIALS AND METHODS}

Melting points of all the chemical compounds and solvents were determined in open capillaries and are uncorrected (Table 1). The IR spectra of all compounds were recorded in FT-IR 8400S Shimadzu spectrophotometer using $\mathrm{KBr}$. Mass spectra were obtained using the 2010EV LCMS Shimadzu instrument at $70 \mathrm{eV}$. ${ }^{1} \mathrm{H}$ NMR spectra were obtained in DMSO on BRUKER AvanceII $400 \mathrm{MHZ}$ instrument, and the chemical shift was measured as parts per million downfield from tetramethylsilane as an internal standard. The target compounds were synthesized as outlined in Scheme 1 .

General method for the synthesis of 2-amino-4,5-diphenyl-1(substituted)-1H-pyrrole-3-carbonitriles (7a-7o)

A mixture of benzoin ( $2 \mathrm{~g}, 0.01 \mathrm{~mol})$, substituted amines $(0.01 \mathrm{~mol})$, and concentrated $\mathrm{HCl}(6-8$ drops $)$ in toluene $(50 \mathrm{ml})$ was heated under reflux for 6 hours and cooled. The reaction mixture was filtered, and the resulting residue was dissolved in $30 \mathrm{ml}$ of absolute alcohol. A malononitrile $(0.66 \mathrm{mg}, 0.01 \mathrm{~mol})$ was added to the previous solution followed by sodium ethoxide ( $2 \mathrm{~g}$ sodium metal in $20 \mathrm{ml}$ of absolute alcohol) as a catalyst. The mixture was refluxed until a solid was formed. The solvent was evaporated under reduced pressure, and the residue was recrystallized from methanol to give the pure yellow crystalline product (Dholakia et al., 2013).

\section{General method for the synthesis of 5,6-diphenyl-7-substituted-} 7H-pyrrolo[2,3-d]pyrimidin-4-yl-amines (8a-8o)

A mixture of each of substituted aminopyrroles $[\mathbf{7 a}-7 \mathbf{0}]$ $(0.01 \mathrm{~mol})$ and formamide $(30 \mathrm{ml}, 0.066 \mathrm{~mol})$ was heated under reflux for 6 hours, cooled, and poured into crush ice to give precipitates which were filtered, dried, and recrystallized from ethanol to yield a yellow crystalline pure product.

General method for the synthesis of 5,6-diphenyl-7-substituted7H-pyrrolo[2,3-d]pyrimidin-4-ols (09a-09o)

A mixture of the substituted aminopyrrole $[7 \mathbf{a}-7 \mathbf{0}](0.01$ mol) and formic acid $(20 \mathrm{ml}, 85 \%)$ was heated under reflux for 3 hours, cooled, and poured onto crush ice to give precipitates which were filtered, dried, and recrystallized from ethanol to yield a yellow crystalline pure product.

General method for the synthesis of 4-chloro-5,6-diphenyl-7substituted-7H-pyrrolo[2,3-d]pyrimidines $(10 a, 10 c, 10 f, 10 m$, 10n, and 10o)

An appropriate pyrrolopyrimid-4-ol $(\mathbf{0 9}, 0.01 \mathrm{~mol})$ in phosphorus oxychloride $(30 \mathrm{ml})$ was heated under reflux for 4 hours, cooled, and poured onto crush ice to give the precipitates which were filtered, dried, and recrystallized from ethanol to yield a yellow crystalline pure product (Gangjee et al., 2010).

\section{General method for cell line study (MTT assay)}

It is a laboratory test and a standard colorimetric assay for measuring cellular growth. It can also be used to determine the cytotoxicity of potential medicinal agents and other toxic materials.

This assay is a sensitive, quantitative, and reliable colorimetric assay that measures viability, proliferation, and activation of cells. The assay is based on the capacity of mitochondrial dehydrogenase enzymes in living cells to convert

Table 1. Physical properties of 2-amino-4,5-diphenyl-1-(substituted)- $1 \mathrm{H}$-pyrrole-3-carbonitriles (7a-7o), 5,6-diphenyl-7-substituted-7Hpyrrolo[2,3- $d$ ] pyrimidin-4-yl-amines (8a-8o), 5,6-diphenyl-7-substituted-7H-pyrrolo[2,3- $d$ ] pyrimidin-4-ols (9a-9o), and 4-chloro-5,6-diphenyl7-substituted-7H-pyrrolo[2,3-d]pyrimidines (10a, 10d, 10f, 10m, 10n, and 10o).

\begin{tabular}{|c|c|c|c|c|c|}
\hline $\begin{array}{c}\text { Comp. } \\
\text { code }\end{array}$ & Melting point $\left({ }^{\circ} \mathrm{C}\right)$ & $\begin{array}{c}\text { Comp. } \\
\text { code }\end{array}$ & Melting point $\left({ }^{\circ} \mathrm{C}\right)$ & $\begin{array}{c}\text { Comp. } \\
\text { code }\end{array}$ & Melting point $\left({ }^{\circ} \mathrm{C}\right)$ \\
\hline $7 a$ & $170-172$ & $8 c$ & $235-237$ & $9 \mathrm{e}$ & $233-235$ \\
\hline $7 b$ & $228-230$ & $8 d$ & $230-232$ & 9f & $210-212$ \\
\hline $7 \mathrm{c}$ & $136-138$ & $8 \mathrm{e}$ & $226-228$ & $9 g$ & $>300$ \\
\hline $7 d$ & $170-172$ & $8 f$ & $260-262$ & $9 \mathrm{~h}$ & $211-211$ \\
\hline $7 e$ & $174-177$ & $8 g$ & $>300$ & $9 \mathrm{i}$ & $260-262$ \\
\hline $7 f$ & $164-166$ & $8 h$ & $273-275$ & $9 \mathrm{j}$ & $>300$ \\
\hline $7 \mathrm{~g}$ & $123-125$ & $8 \mathbf{i}$ & $235-237$ & $9 k$ & $240-242$ \\
\hline $7 \mathrm{~h}$ & $145-147$ & $8 \mathbf{j}$ & $>300$ & 91 & $269-271$ \\
\hline $7 \mathbf{i}$ & $160-122$ & $8 \mathbf{k}$ & $231-333$ & $9 m$ & $280-282$ \\
\hline $7 \mathbf{j}$ & $182-184$ & 81 & $232-234$ & $9 n$ & $>300$ \\
\hline $7 \mathrm{k}$ & $181-183$ & $8 \mathrm{~m}$ & $245-247$ & 90 & $288-290$ \\
\hline 71 & $160-162$ & $8 n$ & $>300$ & $10 \mathrm{a}$ & $>300$ \\
\hline $7 \mathrm{~m}$ & $136-138$ & 80 & $>300$ & 10d & 290-292 \\
\hline $7 n$ & $210-112$ & $9 \mathrm{a}$ & 199-201 & $10 \mathrm{f}$ & $280-282$ \\
\hline 70 & 200-202 & $9 b$ & 213-215 & $10 \mathrm{~m}$ & $>300$ \\
\hline $8 a$ & $262-264$ & $9 \mathrm{c}$ & $180-182$ & $10 \mathrm{n}$ & $>300$ \\
\hline $8 b$ & $218-220$ & 9d & 190-192 & 100 & 291-293 \\
\hline
\end{tabular}


the yellow water-soluble substrate 3-(4,5-dimethylthiazol-2yl)-2,5-diphenyl tetrazolium bromide (MTT) into a dark blue formazan product which is insoluble in water. The amount of formazan produced is directly proportional to the cell number in the range of cell lines (Bruce, 1990; Canavan and Doshi, 2000).

\section{2-amino-1,4,5-triphenyl-1H-pyrrole-3-carbonitrile (7a)}

Yellow crystalline solid, yield $80 \%$, mass $(\mathrm{m} / \mathrm{e}) 336.15$ $(\mathrm{M}+1)$, IR $\left(\mathrm{cm}^{-1}\right) \mathrm{CN}-2,201, \mathrm{NH}_{2}-3,3851 \mathrm{H}, \mathrm{NMR}(\delta \mathrm{ppm}$, DMSO-d6) 5.75 (br-s, 2H, $\mathrm{NH}_{2}$ ), 6.93-7.38 (m, 15H, Ar-H).

2-amino-4,5-diphenyl-1-p-tolyl-1H-pyrrole-3-carbonitrile (7d)

Yellow crystalline solid, yield $60 \%$, mass $(\mathrm{m} / \mathrm{e}) 350.16$ $(\mathrm{M}+1), \mathrm{IR}\left(\mathrm{cm}^{-1}\right) \mathrm{CN}-2,207, \mathrm{NH}_{2}-3,3221 \mathrm{H}, \mathrm{NMR}(\delta \mathrm{ppm}$, DMSO-d6) 2.29 (s, 3H, CH ), 5.70 (br-s, 2H, NH ), 6.93-7.26 (m, $14 \mathrm{H}, \mathrm{Ar}-\mathrm{H})$

\section{5,6,7-triphenyl-7H-pyrrolo[2,3-d]pyrimidin-4-amine (8a)}

Yellow crystalline solid, yield 40\%, mass (m/e) 363.15 $(\mathrm{M}+1)$, IR $\left(\mathrm{cm}^{-1}\right) \mathrm{NH}_{2}-3,4631 \mathrm{H}, \mathrm{NMR}(\delta \mathrm{ppm}, \mathrm{DMSO}-\mathrm{d} 6)$ 8.30 (s, 1H, C-2H), 7.06 (br-s, 2H, NH $)$, 7.15-7.52 (m, 15H, Ar$\mathrm{H})$.

5,6-diphenyl-7-m-tolyl-7H-pyrrolo[2,3-d]pyrimidin-4-amine (8c)

Yellow crystalline solid, yield $60 \%$, mass $(\mathrm{m} / \mathrm{e}) 378.00$ $(\mathrm{M}+1) \mathrm{IR}\left(\mathrm{cm}^{-1}\right) \mathrm{NH}_{2}-3,4881 \mathrm{H}, \mathrm{NMR}(\delta \mathrm{ppm}, \mathrm{DMSO}-\mathrm{d} 6)$ 2.36 (s, 3H, $\left.\mathrm{CH}_{3}\right), 8.33$ (s, 1H, C-2H), 5.02 (br-s, 2H, NH, $\mathrm{D}_{2} \mathrm{O}$ Exchangeable), 6.98-7.40 (m, 14H, Ar-H).

7-(2-chlorophenyl)-5,6-diphenyl-7H-pyrrolo[2,3-d]pyrimidin-4amine (8k)

Yellow crystalline solid, yield 30\%, mass (m/e) 397.12 $(\mathrm{M}+1), 399.11 \mathrm{M}+2 \mathrm{IR}\left(\mathrm{cm}^{-1}\right) \mathrm{NH} 2-3,4651 \mathrm{H}, \mathrm{NMR}(\delta \mathrm{ppm}$, DMSO-d6) 8.33 (s, 1H, C-2H), 6.96-7.38 (m, 14H, Ar-H), 5.11 (br-s, $2 \mathrm{H}, \mathrm{NH}_{2}, \mathrm{D}_{2} \mathrm{O}$ Exchangeable).

\section{5,6,7-triphenyl-7H-pyrrolo[2,3-d]pyrimidin-4-ol (9a)}

Yellow crystalline solid, yield $40 \%$, mass (m/e) 364.00 $(\mathrm{M}+1), \mathrm{IR}\left(\mathrm{cm}^{-1}\right) \mathrm{OH}-3,2411 \mathrm{H}, \mathrm{NMR}(\delta \mathrm{ppm}, \mathrm{DMSO}-\mathrm{d} 6) 8.20$ (s, 1H, C-2H), 10.20 (s, 1H, C-OH), 6.92-7.38 (m, 15H, Ar-H).

\section{5,6-diphenyl-7-m-tolyl-7H-pyrrolo[2,3-d]pyrimidin-4-ol (09c)}

Yellow crystalline solid, yield $60 \%$, mass $(\mathrm{m} / \mathrm{e}) 379.0$ $(\mathrm{M}+1)$, IR $\left(\mathrm{cm}^{-1}\right) \mathrm{OH}-3,2341 \mathrm{H}, \mathrm{NMR}(\delta \mathrm{ppm}, \mathrm{DMSO}-\mathrm{d} 6) 2.34$ $\left(\mathrm{s}, 3 \mathrm{H}, \mathrm{CH}_{3}\right), 8.21(\mathrm{~s}, 1 \mathrm{H}, \mathrm{C}-2 \mathrm{H}), 8.60(\mathrm{~s}, 1 \mathrm{H}, \mathrm{C}-\mathrm{OH}), 6.92-7.37$ (m, 14H, Ar-H).

7-(2-chlorophenyl)-5,6-diphenyl-7H-pyrrolo[2,3-d]pyrimidin-4ol $(9 k)$

Yellow crystalline solid, yield 30\%, mass (m/e) 398.10 $(\mathrm{M}+1), 400.10 \mathrm{M}+2 \mathrm{IR}\left(\mathrm{cm}^{-1}\right) \mathrm{OH}-3,3081 \mathrm{H}, \mathrm{NMR}(\delta$ ppm, DMSO-d6) 7.80 (s, 1H, C-2H), 12.25 (s, 1H, C-OH, D 2 exchangeable), 6.90-7.38 ( $\mathrm{m}, 14 \mathrm{H}, \mathrm{Ar}-\mathrm{H})$.

\section{4-chloro-5,6,7-triphenyl-7H-pyrrolo[2,3-d]pyrimidine (10a)}

Yellow crystalline solid, yield $50 \%$, mass $(\mathrm{m} / \mathrm{e}) 382.11$ $(\mathrm{M}+1), 384.11(\mathrm{M}+2), \mathrm{IR}\left(\mathrm{cm}^{-1}\right) \mathrm{Cl}-6951 \mathrm{H}, \mathrm{NMR}(\delta \mathrm{ppm}$, DMSO-d6) 6.91-7.20 (m,15H, Ar-H), 7.90 (s, 1H, C-2H). 4-chloro-5, 6-diphenyl-7-p-tolyl-7H-pyrrolo[2,3-d]pyrimidine (10d)

Yellow crystalline solid, yield $60 \%$, mass $(\mathrm{m} / \mathrm{e}) 396.11$ $(\mathrm{M}+1), 398.11(\mathrm{M}+2)$, IR $\left(\mathrm{cm}^{-1}\right) \mathrm{Cl}-6971 \mathrm{H}, \mathrm{NMR}(\delta \mathrm{ppm}$, DMSO-d6) 2.51 (s, 3H, CH $), 7.18-7.84$ (m,14H, Ar-H) 8.60 (s, $1 \mathrm{H}, \mathrm{C}-2 \mathrm{H})$

\section{RESULTS AND DISCUSSION}

We synthesized 5,6-diphenyl-7-substituted-7Hpyrrolo[2,3- $d]$ pyrimidin-4-yl-amines (8a-8o), 5,6-diphenyl7-substituted-7H-pyrrolo[2,3- $d]$ pyrimidin-4-ols $\quad$ (9a-9o), and 4-chloro-5,6-diphenyl-7-substituted-7H-pyrrolo[2,3- $d]$ pyrimidines (10a, 10c, 10f, 10m, 10n, and 10o) as depicted in Scheme 1. 2-amino-4,5-diphenyl-1-(substituted)-1H-pyrrole-3carbonitriles $(\mathbf{7} \mathbf{a}-\mathbf{7 0})$ were prepared from the reaction of benzoin with respective amines, followed by the treatment of in situ generated initially formed intermediate $\mathbf{6}$ with malononitrile in the presence of sodium ethoxide. 5,6-Diphenyl-7-substituted7H-pyrrolo[2,3-d]pyrimidin-4-yl-amines $\quad(\mathbf{8 a - 8 0 )} \quad$ were prepared by refluxing 2-amino-4,5-diphenyl-1-(substituted)$1 H$-pyrrole-3-carbonitrile (7a-7o) with formamide. Another series of compounds containing 4-hydroxy group was prepared by refluxing (7a-7o) with formic acid. Some of these 4-hydroxy derivatives $(09 a, 09 c, 09 f, 09 m, 09 n$, and 09o) were converted to the corresponding chloro derivatives $10 \mathrm{a}, 10 \mathrm{c}, 10 \mathrm{f}, 10 \mathrm{~m}$, 10n, and 10o, respectively, by refluxing with phosphorus oxychloride.

In the present investigation, all the compounds were evaluated against cell lines named MDA-MB 468 (Breast cancer cell line) and vero cell line (normal cell line) for each tested compound as well as standard doxorubicin, and the doseresponse curve (DRC) against MDA-MB 468 (Breast cancer cell line) was plotted with 10 analysis point, i.e., with 10 different drug concentrations. The concentration causing 50\% cell growth inhibition $\left(\mathrm{IC}_{50}\right.$ ) was determined from DRC using GraphPad prism software (Ver. 5.04) (GraphPad Software, Inc., La Jolla, CA) and Microsoft Excel 2007 (Microsoft Corporation, Redmond, WA) application (Table 2).

MDA-MB-468 (breast cancer cell line)_-Among all the tested compounds, $8 \mathbf{a}, \mathbf{8 g}, \mathbf{8 h}, \mathbf{8 i}, \mathbf{8 j}, \mathbf{8 k}, \mathbf{8 h}, \mathbf{9 i}, \mathbf{9 j}, \mathbf{9 k}, \mathbf{9 m}$, 9n, 9o, and 10f showed the highest potential effect on MDAMB 468 cell line compared to standard doxorubicin IC50 value. Compounds 8b, 8e, 81, 8m, 8n, 8o, 9a, 9d, 9e, 9f, 9g, 9l, 10a, and 10d possessed a good anticancer/cytotoxicity activity. Compounds 8c, 8d, 8f, 9b, 9c, 10m, 10n, and 100 did not show any activity as their $\mathrm{IC}_{50}$ values are higher than $50 \mu \mathbf{M} / \mathbf{m l}$. All compounds were also checked for their normal cell line activity with vero cell line. Compounds $8 \mathbf{a}, \mathbf{8 b}, \mathbf{8 d}, \mathbf{8 e}, \mathbf{8 h}, \mathbf{8 j}, \mathbf{8 1}, \mathbf{8 m}, \mathbf{8 0}$, 9a, 9c, 9d, 9e, 9f, 9h, 9i, 9j, 9m, 9n, 9o, and 10d were found to be non-toxic to vero normal cell line. Compounds $8 \mathbf{a}, \mathbf{8 h}, \mathbf{8 j}, \mathbf{9 h}$, 9i, 9j, 9m, 9n, and 9o showed a higher anticancer activity than the standard doxorubicin $\mathrm{IC}_{50}$ value on MDA-MB 468 (breast cancer cell line) cell lines with non-toxic to vero normal cell line (Fig. 1). 
Table 2. Cell line study of pyrrolo[2,3- $d]$ pyrimidine analogs.

\begin{tabular}{|c|c|c|c|c|}
\hline \multirow[t]{2}{*}{ Compounds } & \multicolumn{2}{|c|}{ MDA-MB-468 cell line study } & \multicolumn{2}{|c|}{$\begin{array}{l}\text { Vero cell line study } \\
\text { (normal cell line) }\end{array}$} \\
\hline & $\mathbf{I C}_{50}(\mu \mathrm{M} / \mathrm{ml})$ & $R^{2}$ & $\mathbf{I C}_{50}(\mu \mathrm{M} / \mathrm{ml})$ & $R^{2}$ \\
\hline $8 \mathbf{a}$ & 2.72 & 0.9728 & 254.4 & 0.9862 \\
\hline $8 \mathbf{b}$ & 18.09 & 0.9948 & 631 & 0.9891 \\
\hline $8 c$ & 47.2 & 0.9967 & 19.04 & 0.9891 \\
\hline $8 d$ & 28.45 & 0.9925 & $>100$ & 0.9595 \\
\hline $8 \mathrm{e}$ & 14.65 & 0.9754 & $>1,000$ & 0.992 \\
\hline $8 f$ & 37.11 & 0.9653 & 15.72 & 0.9765 \\
\hline $8 \mathrm{~g}$ & 06.57 & 0.9570 & 93.32 & 0.9957 \\
\hline $8 \mathrm{~h}$ & 2.17 & 0.9759 & $>100$ & 0.9738 \\
\hline $8 \mathbf{i}$ & 3.37 & 0.9839 & 8.03 & 0.9767 \\
\hline $8 \mathbf{j}$ & 2.12 & 0.9909 & 143.8 & 0.9778 \\
\hline $8 k$ & 5.4 & 0.9750 & 20.73 & 0.9726 \\
\hline 81 & 12.94 & 0.9628 & 144.92 & 0.9869 \\
\hline $8 \mathrm{~m}$ & 13.11 & 0.9834 & 162 & 0.9946 \\
\hline $8 n$ & 12.15 & 0.9668 & 133 & 0.9933 \\
\hline 80 & 23.69 & 0.9519 & $>1,000$ & 0.9823 \\
\hline 9a & 10.27 & 0.9878 & 209.1 & 0.9986 \\
\hline $9 b$ & 100.8 & 0.9892 & 5.74 & 0.9905 \\
\hline $9 \mathrm{c}$ & 59 & 0.9604 & $>100$ & 0.9764 \\
\hline 9d & 9.51 & 0.9987 & 144.9 & 0.9978 \\
\hline $9 e$ & 23.05 & 0.9884 & $>100$ & 0.9553 \\
\hline 9f & 21.11 & 0.9842 & 487.4 & 0.9822 \\
\hline $9 \mathrm{~g}$ & 11.55 & 0.9693 & 6.9 & 0.9606 \\
\hline $9 \mathrm{~h}$ & 7.24 & 0.9564 & $>1,000$ & 0.9955 \\
\hline $9 \mathbf{i}$ & 5.79 & 0.9449 & 299 & 0.8944 \\
\hline $9 j$ & 4.23 & 0.9762 & $>100$ & 0.9988 \\
\hline $9 k$ & 4.93 & 0.9556 & 79.71 & 0.9902 \\
\hline 91 & 11.79 & 0.9535 & 17.06 & 0.9783 \\
\hline $9 m$ & 2.05 & 0.9897 & $>100$ & 0.9798 \\
\hline $9 n$ & 2.11 & 0.9838 & 410.4 & 0.9923 \\
\hline 90 & 4.2 & 0.9617 & 257.5 & 0.9948 \\
\hline $10 \mathrm{a}$ & 9.66 & 0.9899 & $>100$ & 0.9645 \\
\hline $10 \mathrm{c}$ & 25.39 & 0.9891 & 94.89 & 0.9908 \\
\hline $10 \mathrm{f}$ & 4.62 & 0.9692 & 23.84 & 0.9625 \\
\hline $10 \mathrm{~m}$ & 247.9 & 0.9741 & 57.06 & 0.9911 \\
\hline $10 n$ & 89.04 & 0.9955 & 69.66 & 0.9755 \\
\hline $10 \mathrm{o}$ & 100 & 0.9736 & 24.43 & 0.9044 \\
\hline Std. & 6.17 & 0.9229 & 314.6 & 0.9921 \\
\hline
\end{tabular}

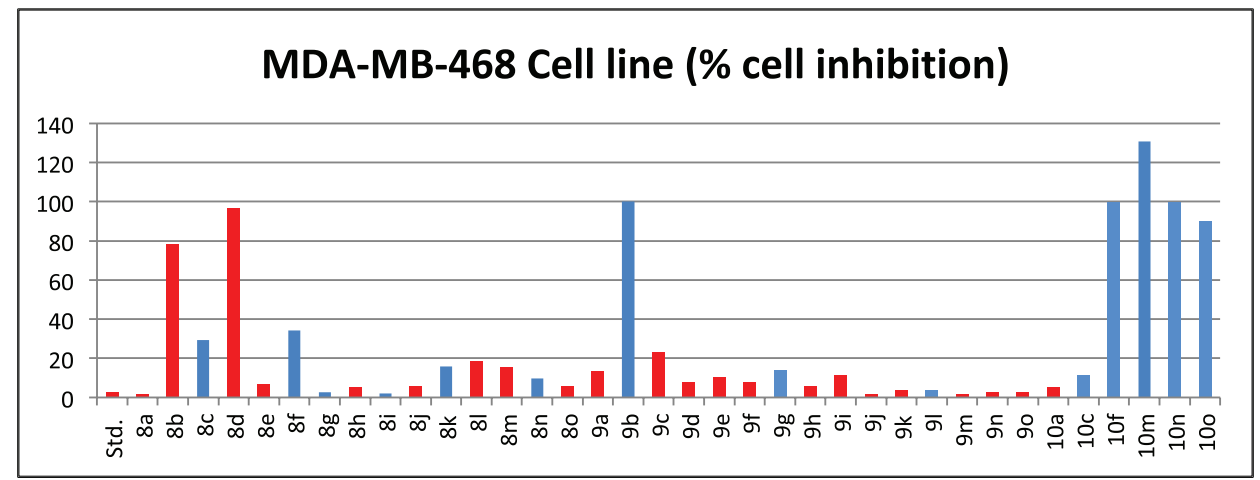

Figure 1. Graphical representation of cell line activity of pyrrolo[2,3- $d$ ]pyrimidine analogs.

(Blue line indicates that the compounds are toxic to vero normal cell line, and the red line indicates that the compounds are non-toxic to vero normal cell line) against standard doxorubicin. 


\section{CONCLUSION}

From this cell line study, it can be concluded that compounds series with electron-withdrawing groups on substituent at N7 and electron-donating groups on substituent at $\mathrm{C} 4$ give the excellent cytotoxic activity. The compounds with electrondonating or no groups on substituent at N7 and electron-donating groups on substituent at $\mathrm{C} 4$ give a good cytotoxic activity, whereas compounds containing electron-withdrawing groups on substituent at $\mathrm{N} 7$ and $\mathrm{C} 4$ do not give the cytotoxic activity on MDA-MB 468 (breast cancer cell line).

\section{CONFLICT OF INTEREST}

Authors declare that they do not have any conflicts of interest.

\section{FUNDING}

None.

\section{REFERENCES}

Bruce AC. In defense of cell line screening. J Natl Cancer Inst, 1990; 82(13):1107-12.

Canavan TP, Doshi NR. Cervical cancer. Am Fam Phys, 2000; 61(5):1369-76.

Dholakia SP, Patel SA. Synthesis and antimicrobial screening of novel 2-amino-4,5-Diphenyl-1-(Substituted)-1H-Pyrrole-3-Carbonitrile. Am J PharmTech Res, 2013; 3(5):447-52.

Edge SB, Byrd DR, Compton CC, Fritz AG, Greene FG, Trotti A. AJCC cancer staging manual. Springer Publisher, New York, NY, 2010.

Foye WO, Lemke TL, William DA. Principles of medicinal chemistry USA. Lippincott Williams \& Wilkins Publisher, Philadelphia, PA, 1995.

Gangjee A, Kurup S, Ihnat MA, Thorpe JE, Shenoy SS. Synthesis and biological activity of $N^{4}$-phenylsubstituted-6-(2,4dichlorophenylmethyl)-7 $H$-pyrrolo[2,3- $d]$ pyrimidine-2,4-diamines as vascular endothelial growth factor receptor- 2 inhibitors and antiangiogenic and antitumor agents. Bioorg Med Chem, 2010; 18(10):3575-87.
Ghorab MM, Ragab FA, Alqasoumi SI, Alafeefy AM, Aboulmagd SA. Synthesis of some new Pyrrolo[2,3- $d$ ]pyrimidine and pyrazolo[3,4- $d]$ pyrimidine derivatives of expected anticancer and radioprotective activity. Eur J Med Chem, 2010; 45:171-8.

Grange JM, Stanford JL, Stanford CA, Morgan CD. Observations on cancer and their relevance today. J R Soc Med, 2002; 6:296-302.

Hadfield JA, Ducki S, Hirst N, McGown AT. Tubulin and microtubules as targets for anticancer drugs. Prog Cell Cycle Res, 2003; 5:309-25.

Honore, S, Pasquier E, Braguer D. Understanding microtubule dynamics for improved cancer therapy. Cell Mol Life Sci, 2005; 62:3039-56.

Jordan A, Hadfield JA, Lawrence NJ, McGown AT. Tubulin as a target for anticancer drugs: agents which interact with the mitotic spindle. Med Res Rev, 1998; 18:259-96.

Kari N, Dave W. Rediscovering biology: molecular to global perspectives. Portland Publisher, London, UK, 2012.

Kleinsmith LJ. Principles of cancer biology. Pearson International Publisher, New York, NY, 2006.

Miller KD, Siegel RL, Lin CC. Cancer treatment and survivorship statistics. Cancer J Clin, 2016; 66:271-89.

Whitman W, Coleman D, Wiebe W. Prokaryotes: the unseen majority. Proc Natl Acad Sci, 1995; 12:6578-83.

How to cite this article:

Dholakia SP, Kanada KB, Parmar GR. Synthesis and biological evaluation of novel substituted pyrrolo[2,3-d] pyrimidines as anticancer agents. J Adv Vet Anim Res 2020; 10(06):006-011. 